\title{
Seasonal variations in blood culture numbers and time to positivity and potential impact of reducing incubation periods
}

\author{
Gauthier Péan de Ponfilly ${ }^{1,2}$ (D) Julie Lourtet-Hascoet ${ }^{2} \cdot$ Huong Porcheret $^{3} \cdot$ Emmanuelle Cambau $^{1}$. \\ Alban Le Monnier $^{2} \cdot$ Hervé Jacquier ${ }^{1} \cdot$ Marc Vasse $^{4} \cdot$ Eric Farfour $^{4}$
}

Received: 8 September 2020 / Accepted: 11 April 2021 / Published online: 23 April 2021

(C) The Author(s), under exclusive licence to Springer-Verlag GmbH Germany, part of Springer Nature 2021

\begin{abstract}
Patients with viral respiratory infections often present symptoms compatible with bloodstream infections. Consequently, the winter period commonly associated with epidemic respiratory illnesses shows an increase in the number of blood cultures (BC) and to occasional saturation of automated $\mathrm{BC}$ systems. Here, we explored the seasonal variations in $\mathrm{BC}$ samples and the potential impact of shortening the incubation time of $\mathrm{BC}$ when automated $\mathrm{BC}$ systems are close to saturation. A retrospective study was conducted during a 3-year period in 4 hospitals located in the Paris region, France. All aerobic and anaerobic bottles were included, except pediatric bottles and those sampled for suspicion of endocarditis. The number of BC bottles collected during the winter period was compared to the annual baseline. All bottles positive after a 4-day incubation were analyzed regarding clinical and microbiological findings. The number of $\mathrm{BC}$ bottles was significantly higher during the winter periods, compared to the annual baseline (up to 14\%). A total of 292,349 BC bottles were analyzed with 23,363 (8.0\%) positive, including $236(1 \%)$ after a 4-day incubation. Of these 236 bottles, $76(64.8 \%)$ were positive with a contaminant, $78(33.1 \%)$ with a clinically significant microorganism identified for the same patient in the previous 4 days, and only $5(2.1 \%)$ with a clinically significant microorganism not previously identified. Winter periods were associated with a significant increase in BC samples. Shortening the incubation time of $\mathrm{BC}$ bottles from 5 to 4 days seems a relevant option when automated $\mathrm{BC}$ systems are close to saturation.
\end{abstract}

Keywords Blood culture $\cdot$ Time-to-positivity (TTP) $\cdot$ Influenza $\cdot$ Outbreak $\cdot$ Pandemic

\section{Introduction}

The winter influenza epidemic is associated with an increased number of attendances in the emergency departments, hospitalizations, and an excess of mortality [1]. These patients often

Marc Vasse and Eric Farfour contributed equally to this work.

Gauthier Péan de Ponfilly

gpeandeponfilly@ghpsj.fr

1 Département des Agents Infectieux, Laboratoire de Bactériologie, CHU Saint Louis-Lariboisière-Fernand Widal, APHP, 75010 Paris, France

2 Service de Microbiologie Clinique et Plateforme de dosage des anti-infectieux, Groupe Hospitalier Paris Saint Joseph, 75014 Paris, France

3 Laboratoire de Biologie, Centre Hospitalier Robert Ballanger (CHRB), 93602, Aulnay-Sous-Bois, France

4 Service de Biologie Clinique, Hôpital Foch, 92150 Suresnes, France present symptoms such as undetermined fever $\left(\geq 38^{\circ} \mathrm{C}\right)$ or raised respiratory rate, compatible with bloodstream infections (BSI). Consequently, the winter period shows an increased number of blood cultures sampled and may be associated with a risk of saturation of automated blood culture systems. For blood cultures, a bottle must be removed from the automated device to load another. If the number of bottles exceeds the capacities of the automated device, their incubation would be delayed. Several strategies such as reducing the number of bottles sampled by patients, delaying the incubation of blood culture bottles, or processing to a manual incubation of the bottles could be proposed. However, all of them have limitations and might reduce the performance of blood culture analysis. The median time-to-positivity (TTP) of positive blood culture was previously reported to be less than $24 \mathrm{~h}$ for most microorganisms [2-4]. Although the recommended incubation time of blood culture bottles is 5 days, shortening incubation time is a possible solution. Most of the previous studies focused on the median TTP, but the clinical relevance of late-positivity bottles has rarely been explored [2-4]. 
Therefore, the potential impact of shortening the incubation time of blood culture bottles should be assessed before implementing such a strategy, with particular emphasis on the clinical relevance of late-positivity bottles.

Here we performed a 3-year multicenter study to (1) explore seasonal quantitative variations in blood cultures and (2) to determine the time to positivity of blood culture bottles and the clinical relevance of late-positivity bottles. Altogether, these observations could provide well-documented information to shorten incubation time of $\mathrm{BC}$ during periods with a risk of saturation of automated devices.

\section{Material and methods}

\section{Participant center and blood culture bottles}

We performed a multicenter retrospective study between August 1, 2015, and July 31, 2018. Four hospitals located in the Paris region (France) participated in the study: Foch Hospital (Suresnes) (center A), Robert Ballanger Hospital (Aulnay-Sous-Bois) (center B), Lariboisière University Hospital (Paris) (center C), and Paris Saint-Joseph Hospital (Paris) (center D). All blood cultures collected on aerobic and anaerobic bottles were included, and pediatric bottles were excluded. Automated devices for blood culture incubation were BacT/ALERT (BioMérieux, Marcy-l'étoile, France) for centers $\mathrm{C}$ and D and BactecFX (Becton Dickinson Franklin Lakes, NJ, USA) for centers A and B. According to International Guidelines, 4 blood culture bottles should be sampled in each patient with suspected bacteremia [5]. The blood culture bottles were processed in the automated device 7 days a week during opening hours. They were incubated for 5 days as recommended by the manufacturer and French guidelines [6]. Blood culture bottles incubated for more than 5 days (i.e., sampled in a context of suspicion of endocarditis) were excluded. The TTP was defined as the time between the start of incubation and the detection of bacterial growth by the automated system.

Gram staining was performed for all positive blood culture bottles. One drop of broth was inoculated on blood agar plates under anaerobic condition and Polyvitex chocolate agar plate under $5 \%$ of $\mathrm{CO}_{2}$ for a 2-day incubation at $35^{\circ} \mathrm{C}$. All culture media were supplied by BioMérieux (Marcy-L'Etoile, France). Growing colonies were identified using a Microflex LT mass spectrometer and the MBT Compass software (Bruker Daltonics, Bremen, Germany).

\section{Seasonal variation in the number of blood culture bottles}

The variation of blood culture bottles incubated was calculated and graphed by month and by center. The seasonal evolution in the number of blood culture bottles incubated was calculated for each 1-year period. A 1-year period was centered on the 4 "winter" months (i.e., 1 December to 31 March) and was defined from August 1 to July 31). The annual baseline was defined as the average of the monthly number of blood cultures collected over the 1-year period under consideration, excluding the winter months. Each month was compared to the annual baseline for that year, and the percentage change was reported.

\section{Assessing the impact of a 4-day incubation}

All positive bottles incubated between August 1, 2015, and July 31, 2018 were included. The TTP was analyzed by type of bottles and classed by slots of $6 \mathrm{~h}$. All blood cultures positive for 4 or more days following incubation were analyzed regarding clinical and microbiological findings [7, 8]. A microorganism responsible for infection was considered as "clinically significant," in contrast to a "contaminant," according to the European Society of Clinical Microbiology and Infectious Diseases guidelines [9].

The presence of one of the following microorganisms in a single $\mathrm{BC}$ bottle or $\mathrm{BC}$ set was a priori considered as contaminant: coagulase-negative staphylococci (CoNS), with the exception of $S$. lugdunensis, Propionibacterium spp., Bacillus spp. other than B. anthracis, Corynebacterium spp. (diphtheroids), Aerococcus-like organisms, Micrococcus spp., viridans group streptococci other than $S$. pneumoniae, and Neisseria spp. other than $N$. gonorrhoeae or N. meningitidis.

These microorganisms were considered as significant when other BC bottles collected $48 \mathrm{~h}$ before were positive with the same microorganism, after reviewing of clinical data.

In case of "clinically significant" microorganism detected, results of all other bottles sampled within 4 days were reviewed, and then the positive bottle was classed between "clinically significant with anteriority" and "clinically significant without anteriority."

\section{Statistical analysis}

For the statistical comparison, a Shapiro-Wilk test for normality of distribution was first performed, and a $t$-student test was performed for continuous variables, using the $\mathrm{R}$ software version 3.1.3 [10]. Significance was considered if $p<0.05$.

\section{Ethics}

The study was carried out in accordance with the Declaration of Helsinki. This study was a non-interventional study with no addition to standard care. Biological material and clinical data were obtained only for standard diagnostic following physicians' prescriptions (no specific sampling, no modification of 
the sampling protocol). Data analyses were carried out using an anonymized database.

\section{Results}

\section{Seasonal variation of the number of blood culture bottles}

Overall, the number of processed blood culture bottles was significantly higher during the winter periods, compared to the annual baseline (Fig. 1). This relative increase was $14.2 \%, 12.1 \%$, and $12.8 \%$ for winter periods $2015-2016$, 2016-2017, and 2017-2018, respectively $(p<0.05)$.

The magnitude of the change was variable depending on the center and the winter (Fig. 2). Center A encountered the highest increase in all winter periods, while the increase was below 5\% for each winter period in center C. Center A experienced each year a significant increase in all 4 months of winter, and a similar trend is noticed in center $\mathrm{D}$. In center $\mathrm{C}$ and $\mathrm{D}$, the increase was heterogeneous between and within each.

\section{Significance of blood culture bottles with TTP greater than 4 days}

A total of 292,349 blood culture bottles, including 146,581 aerobic and 145,768 anaerobic bottles, were processed during the study period bottles (Table 1). The rate of positivity was significantly higher in aerobic $(12,318 / 146,581 ; 8.4 \%)$ than in anaerobic bottles $(11,045 / 145,768 ; 7.6 \%)(p<0.01)$.

Growth was detected for $83.6 \%, 95.2 \%, 98.2 \%$, and $99.4 \%$ of positive aerobic bottles after $24 \mathrm{~h}, 48 \mathrm{~h}, 72 \mathrm{~h}$, and $96 \mathrm{~h}$ of incubation, respectively (Supplementary Table 1). Growth was detected for $75.9 \%, 93.5 \%, 97.2 \%$, and $98.6 \%$ of positive anaerobic bottles after $24 \mathrm{~h}, 48 \mathrm{~h}, 72 \mathrm{~h}$, and $96 \mathrm{~h}$ of incubation, respectively (Supplementary Table 1 ).

As the cumulative positivity rate was high after a 4-day incubation, we then focused on the clinical relevance of blood culture bottles with TTP greater than 4 days.

Out of 23,363 positive bottles, $236(1.0 \%)$ were positive following 4 days of incubation. All microorganisms were bacteria, no yeast grown following 4 days of incubation. Considering the 12,318 positive aerobic bottles, $86(0.70 \%)$ were positive after an incubation time exceeding 4 days. In total, 39 (45.3\%), $43(50.0 \%)$, and 4 (4.7\%) were considered as "contaminant," "clinically significant with anteriority," and "clinically significant without anteriority," respectively. The contaminant microorganisms were coagulase-negative staphylococci (S. pettenkoferi and S. hominis), Corynebacterium species (Corynebacterium minutissimum and Corynebacterium spp.), Cutibacterium acnes, Curtobacterium spp., and Micrococcus luteus.

Of the 11,045 positive anaerobic bottles, $150(1.4 \%)$ were positive following 4 days of incubation. Of those positive bottles, 114 (76.0\%) were considered as contaminated, almost exclusively with $C$. acnes, except one with $S$. epidermidis. The other bottles were positive to a microorganism considered as clinically significant in patients with $(n=35 ; 23.2 \%)$ and without $(n=1 ; 0.7 \%)$ another bottle positive for the same microorganisms in the previous 4 days.

Overall, $5(0.002 \%)$ blood culture bottles were positive with a clinically significant microorganism without anteriority in the first 4 days of incubation (Table 2). Except Streptococcus gallolyticus, all were fastidious microorganisms: Alloscardovia omnicolens $(n=1)$, Campylobacter upsaliensis $(n=1)$, and Capnocytophaga canimorsus $(n=1)$ and $C$. acnes $(n=1)$. The TTP of these bottles ranges from 110 to $119 \mathrm{~h}$. In two cases, another clinical sample was positive with the same microorganism, a urinary tract infection
Fig. 1 Overall seasonal variation in the number of blood-culture bottles incubated in automated system. A year period begins from August 1 to July 31 . Months of winter are represented by red bars and other months by blue bars

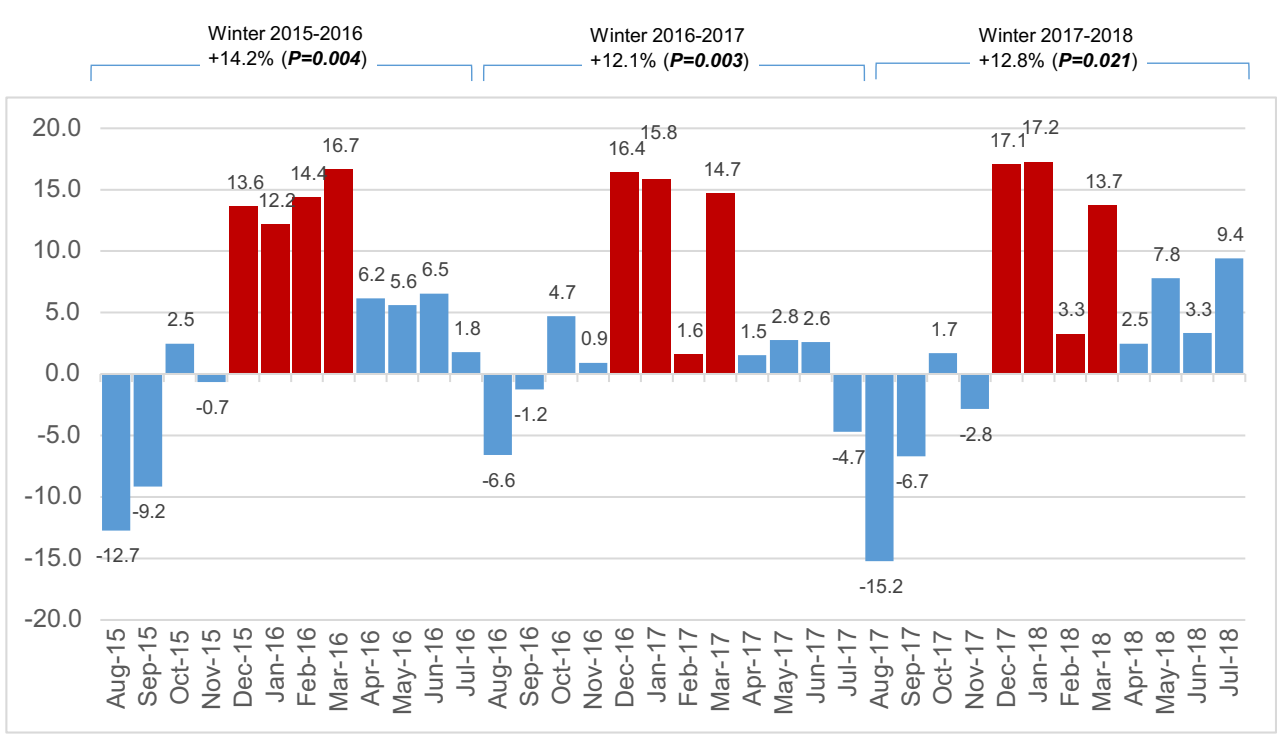



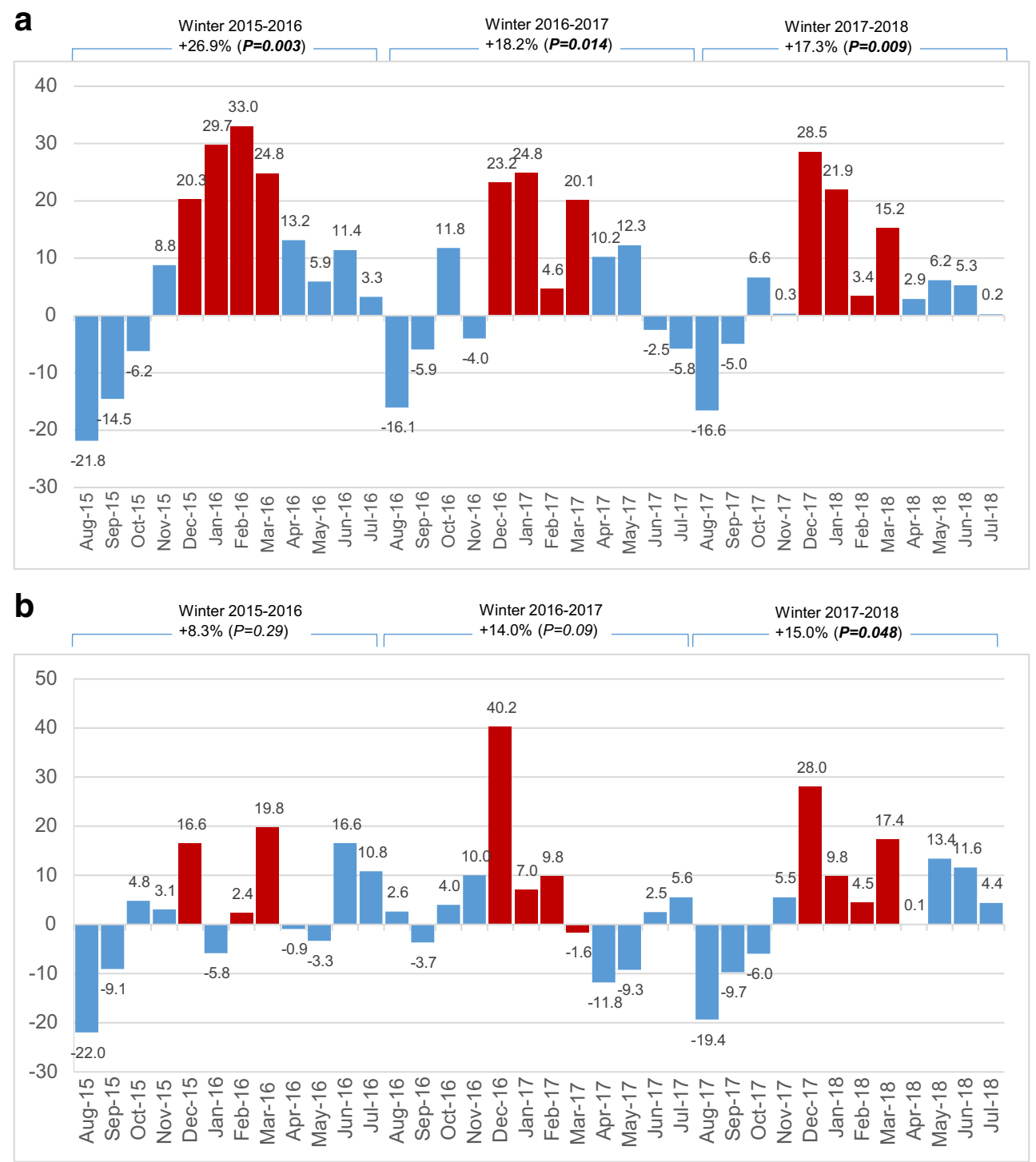

Fig. 2 Seasonal variation in the number of blood-culture bottles incubated in automated system by center. a Results for center A, b results for center $\mathrm{B}, \mathbf{c}$ results for center $\mathrm{C}, \mathbf{d}$ results for center $\mathrm{D}$. A year period begins

due to Alloscardovia omnicolens and a cervical cellulitis due to Cutibacterium acnes. In two cases, an effective antibiotic therapy was administered before the positivity of the blood culture.

\section{Discussion}

Our results demonstrate a seasonal variation in the number of blood culture bottles incubated in automated devices, with an increase during winter period. If automated devices are overwhelmed, we suggest shortening the duration of incubation has no impact on the sensitivity of bacteremia diagnosis for most patients. from August 1 to July 31. Months of winter are represented by red bars and other months by blue bars

The increase in the number of blood culture bottles incubated in winter might be related to the influenza outbreak which is associated with an increase in consultation, hospitalization, and an excess of death [1]. However, the variation appears heterogeneous regarding each hospital and different winter season.

As this increased activity could lead to a saturation of automated devices, several strategies are possible to face this situation. First, it is possible to delay the incubation of blood culture bottles. However, time-to-results would be increased, and consequently, the medical management of the patients worsened $[5,11]$. Furthermore, this option would only respond to a brief increase in the number of blood cultures bottles, which does not correspond to an epidemic situation. Indeed, it would lead to a 

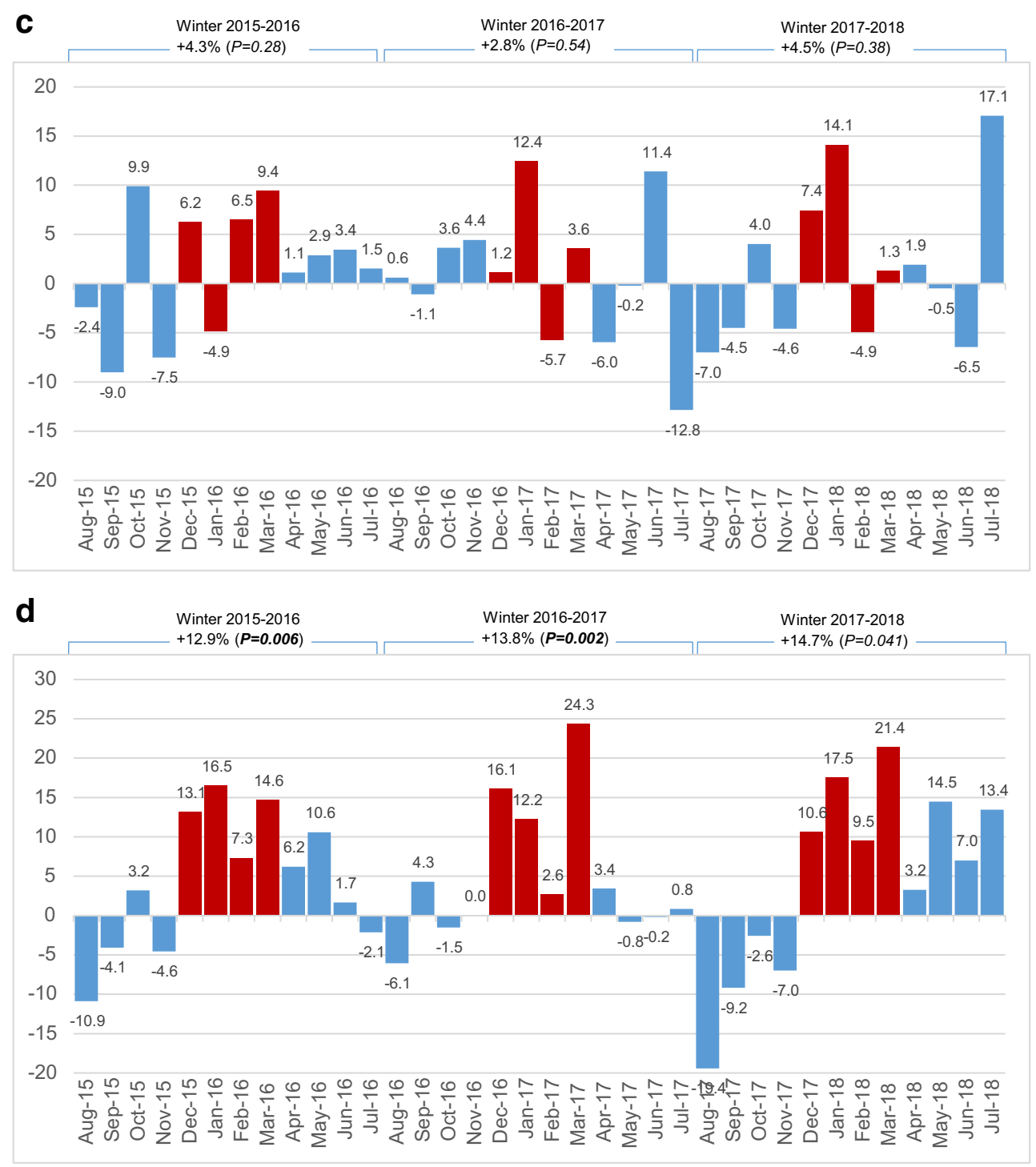

Fig. 2 (continued)

continuous increase in the number of bottles awaiting incubation. Then, incubating blood culture bottles in a laboratory oven with daily plating on agar media might allow increasing significantly the number of blood culture bottles processed. However, it is time-consuming and associated with decreased sensitivity and specificity $[12,13]$. Otherwise, it is possible to reduce the number of bottles sampled by patients, but the sensitivity of the analysis might also be negatively impacted. Finally, reducing the duration of incubation is probably the simplest and quickest way to solve this situation. However, the major risk of this strategy is to reduce the sensitivity of the blood culture by increasing the rate of false negative.

The median TTP was previously reported as less than $24 \mathrm{~h}$ for a wide range of microorganisms [2-4, 14], but extreme
Table 1 Clinical interpretation of positive blood cultures in more than 4 days

\begin{tabular}{llll}
\hline & Aerobic $(n=146,581)$ & Anaerobic $(n=145,768)$ & $\begin{array}{l}\text { Overall } \\
(n=292,349)\end{array}$ \\
\hline Positive bottles $(n ; \%)$ & $12,318(8.4 \%)$ & $11,045(7.6 \%)$ & $23,363(8.0 \%)$ \\
Time-to-positivity $>4$ days $(n ; \%)$ & $86(0.7 \%)$ & $150(1.4 \%)$ & $236(1.0 \%)$ \\
Contaminant $(\%)$ & $39(45.3 \%)$ & $114(76.0 \%)$ & $153(64.8 \%)$ \\
Anteriority positive $(\%)$ & $43(50.0 \%)$ & $35(23.3 \%)$ & $78(33.1 \%)$ \\
Significant $(\%)$ & $4(4.7 \%)$ & $1(0.7 \%)$ & $5(2.1 \%)$ \\
\hline
\end{tabular}


Table 2 Characteristics of the patients displaying a positive blood culture bottles in more than 4 days due to a "clinically significant" microorganism

\begin{tabular}{|c|c|c|c|c|c|c|}
\hline Patient $n^{\circ}$ & $\begin{array}{l}\text { Type of } \\
\text { bottle }\end{array}$ & TTP (hours) & Species & $\begin{array}{l}\text { Other clinical sample } \\
\text { positive to the same } \\
\text { microorganism }\end{array}$ & $\begin{array}{l}\text { Effective antibiotic therapy } \\
\text { before positivity of blood culture }\end{array}$ & Diagnosis \\
\hline 1 & Aerobic & 110 & Streptococcus gallolyticus & No & Yes & Digestive translocation \\
\hline 2 & Aerobic & 119 & Alloscardovia omnicolens & Yes (urine) & No & Urinary tract infection \\
\hline 3 & Aerobic & 117 & Campylobacter upsaliensis & No & Yes & Digestive infection \\
\hline 4 & Aerobic & 113 & Capnocytophaga canimorsus & No & No & Pneumonia \\
\hline 5 & Anaerobic & 116 & Cutibacterium acnes & Yes (surgical sample) & No & Cellulitis \\
\hline
\end{tabular}

TTP was rarely reported, and therefore, the impact of shortening the duration of incubation on the sensitivity on the diagnosis of bacteremia was unclear [15]. Recently, using a Bactec FX automated device, Sepulveda et al. reported that $98.2 \%$ of 1859 positive blood culture bottles during the COVID-19 pandemic were positive in less than 4 days [16]. However, the authors did not distinguish aerobic and anaerobic bottles, they do not explore positive bottles in more than 4 days, and the number of bottles included was 10 times lower than in the present study. In another study, Marginson et al. showed that $2.7 \%$ of all positive blood culture was positive after 5 days of incubation, including $0.5 \%$ of clinically relevant organisms [17]. Our results highlight that most bottles positive in more than 4 days are due to "contaminant," and for "clinically significant" microorganisms, another bottle is frequently positive to the same microorganism with a shorter TTP. Consequently, the risk to dismiss bacteremia when shortening the duration of incubation is null in most patients for common pathogens using both the BactecFX and the BactAlert devices. If the increase in number of bottles processed is limited, the strategy could be implemented to a single type of bottles. In our experience, a higher rate of anaerobic bottles turns positive following 4 days of incubation, but most of them grow a "contaminant" microorganism mainly $C$. acnes, suggesting that reducing the duration of incubation of this single type of bottle is probably more accurate. However, the TTP might vary depending on several factors, and we show that a patient could display a positive bottle with TTP greater than 4 days and another lower than 4 days. Therefore, accurate practices of blood culture sampling (i.e., number of bottles sampled and volume of blood) are required before shortening the duration of incubation. Moreover, this strategy should not be implemented when a fastidious growing microorganism is suspected, e.g., in the context of endocarditis, even if several studies showed that prolonged incubation time does not significantly improve the overall sensitivity of blood culture [18]. Furthermore, in case of suspicion of candidemia, previous reports suggests a TTP superior to $96 \mathrm{~h}$, especially for Candida glabrata $[19,20]$.

Our study has several limitations. First, as we assess the number of bottles incubated each month, a sudden increase in the number of blood cultures collected over several days can lead to saturation of the automated device and will not be visible in the monthly statistics. Second, this is a retrospective study with the inherent biases of this type of method. Finally, we did not have data on the number of solitary blood cultures collected (defined as a single BC set sampled in a 24-h period). Indeed, it is now well established that the volume of blood drawn is an important factor in the positivity rate of blood cultures [21, 22].

Few pediatric bottles are sampled in our hospitals, and we were not able to assess the impact of shortening the duration of incubation of these bottles. But a previous report suggests that the TTP of positive pediatric bottles could reach up to $96 \mathrm{~h}$; consequently, shortening the incubation of these bottles should be assessed before implementation [23].

In conclusion, an adaptive strategy is required when blood culture-automated devices are overwhelmed in the context of an infectious epidemic. As most blood culture bottles turn positive in less than 4 days, shortening the duration of incubation appears the most relevant solution in order to free additional capacity. However, TTP might vary according to several factors such as local microbial epidemiology or practice in blood culture sampling. We, therefore, suggest that this strategy should be validated in each laboratory before implementation. We also suggest that accurate practices of blood culture sampling are required even more in the particular context of an infectious epidemic.

Supplementary Information The online version contains supplementary material available at https://doi.org/10.1007/s10096-021-04248-9.

\section{Declarations}

Conflict of interest The authors declare no competing interest.

\section{References}

1. Pivette M, Nicolay N, Lauzun V, Hubert B (2020) Characteristics of hospitalizations with an influenza diagnosis, France, 2012-2013 to 2016-2017 influenza seasons. Influenza Other Respir Viruses 5;irv.12719. https://doi.org/10.1111/irv.12719 
2. Kirn TJ, Mirrett S, Reller LB, Weinstein MP (2014) Controlled clinical comparison of BacT/alert FA plus and FN plus blood culture media with BacT/alert FA and FN blood culture media. J Clin Microbiol 52:839-843. https://doi.org/10.1128/JCM.03063-13

3. Lee DH, Kim SC, Bae IG, Koh EH, Kim S (2013) Clinical evaluation of bacT/Alert FA plus and FN plus bottles compared with standard bottles. J Clin Microbiol 51:4150-4155. https://doi.org/ 10.1128/JCM.01935-13

4. Fiori B, D'Inzeo T, Di Florio V, De Maio F, De Angelis G, Giaquinto A et al (2014) Performance of two resin-containing blood culture media in detection of bloodstream infections and in direct matrix-assisted laser desorption ionization-time of flight mass spectrometry (MALDI-TOF MS) broth assays for isolate identification: clinical comparison of the BacT/Alert Plus and Bactec Plus systems. J Clin Microbiol 52(1):3558-3567. https:// doi.org/10.1128/JCM.01171-14

5. Rhodes A, Evans LE, Alhazzani W, Levy MM, Antonelli M, Ferrer $R$ et al (2017) Surviving Sepsis Campaign: International Guidelines for Management of Sepsis and Septic Shock: 2016. Intensive Care Med 43(1):304-377. https://doi.org/10.1007/s00134-017-4683-6

6. Société Française de Microbiologie(2019) Hémoculture. In: REMIC 6.1

7. Pien BC, Sundaram P, Raoof N, Costa SF, Mirrett S, Woods CW et al (2010) The clinical and prognostic importance of positive blood cultures in adults. Am J Med 123:819-828. https://doi.org/ 10.1016/j.amjmed.2010.03.021

8. Leber AL (ed) (2019) Clinical Microbiology Procedures Handbook, 4th edn

9. Doern GV, Carroll KC, Diekema DJ, Garey KW, Rupp ME, Weinstein MP et al (2019) Practical guidance for clinical microbiology laboratories: a comprehensive update on the problem of blood culture contamination and a discussion of methods for addressing the problem. Clin Microbiol Rev 33(18):e00009-e00019. https://doi.org/10.1128/CMR.00009-19

10. R Core Team (2020) R: a language and environment for statistical computing [Internet]. R Foundation for Statistical Computing, Vienna, Austria. https://www.r-project.org

11. Kumar A, Roberts D, Wood KE, Light B, Parrillo JE, Sharma S et al (2006) Duration of hypotension before initiation of effective antimicrobial therapy is the critical determinant of survival in human septic shock. Crit Care Med 34:1589-1596. https://doi.org/10. 1097/01.CCM.0000217961.75225.E9

12. Rohner P, Pepey B, Auckenthaler R (1995) Comparison of BacT/ Alert with signal blood culture system. J Clin Microbiol 33(1):313317. https://doi.org/10.1128/jcm.33.2.313-317.1995

13. Rohner P, Pepey B, Auckenthaler R (1996) Comparative evaluation of BACTEC aerobic Plus/F and Septi-Chek Release blood culture media. J Clin Microbiol 34(1):126-129

14. Zadroga R, Williams DN, Gottschall R, Hanson K, Nordberg V, Deike $M$ et al (2013) Comparison of 2 blood culture media shows significant differences in bacterial recovery for patients on antimicrobial therapy. Clinical infectious diseases: an official publication of the Infectious Diseases Society of America 56:790-797. https://doi.org/10.1093/cid/cis1021

15. Bourbeau PP, Pohlman JK (2001) Three days of incubation may be sufficient for routine blood cultures with BacT/Alert FAN blood culture bottles. J Clin Microbiol 39:2079-2082. https://doi.org/10. 1128/JCM.39.6.2079-2082.2001

16. Sepulveda J, Westblade LF, Whittier S, Satlin MJ, Greendyke WG, Aaron JG et al (2020) Bacteremia and blood culture utilization during COVID-19 surge in New York City. J Clin Microbiol 13: 58. https://doi.org/10.1128/jcm.00875-20

17. Marginson MJ, Daveson KL, Kennedy KJ (2014) Clinical impact of reducing routine blood culture incubation time from 7 to 5 days. Pathology 46:636-639. https://doi.org/10.1097/PAT. 0000000000000167

18. Petti CA, Bhally HS, Weinstein MP, Joho K, Wakefield T, Reller LB et al (2006) Utility of extended blood culture incubation for isolation of Haemophilus, Actinobacillus, Cardiobacterium, Eikenella, and Kingella organisms: a retrospective multicenter evaluation. J Clin Microbiol 44:257-259. https://doi.org/10.1128/JCM. 44.1.257-259.2006

19. Huang L, Zhang YY, Sun LY (2013) Time to positivity of blood culture can predict different Candida species instead of pathogen concentration in candidemia. Eur J Clin Microbiol Infect Dis 32: 917-922. https://doi.org/10.1007/s10096-013-1826-8

20. Cobos-Trigueros N, Kaasch AJ, Soriano A, Torres J-L, Vergara A, Morata L et al (2014) Time to positivity and detection of growth in anaerobic blood culture vials predict the presence of candida glabrata in candidemia: a two-center European cohort study. J Clin Microbiol 52:3082-3084. https://doi.org/10.1128/JCM. 01198-14

21. Schifman RB, Strand CL, Braun E, Louis-Charles A, Spark RP, Fried ML (1991) Solitary blood cultures as a quality assurance indicator. Qual Assur Util Rev 6:132-137. https://doi.org/10. 1177/0885713x9100600406

22. Dargère S, Parienti J-J, Roupie E, Gancel P-E, Wiel E, Smaiti N et al (2014) Unique blood culture for diagnosis of bloodstream infections in emergency departments: a prospective multicentre study. Clin Microbiol Infect 20:O920-O927. https://doi.org/10. 1111/1469-0691.12656

23. Doern CD, Mirrett S, Halstead D, Abid J, Okada P, Reller LB (2014) Controlled clinical comparison of new pediatric medium with adsorbent polymeric beads (PF Plus) versus charcoalcontaining PF medium in the bact/alert blood culture system. J Clin Microbiol 52:1898-1900. https://doi.org/10.1128/JCM. 00175-14

Publisher's note Springer Nature remains neutral with regard to jurisdictional claims in published maps and institutional affiliations. 